\title{
Kinetics of 5-Aminolevulinic Acid-Induced Fluorescence in Organ Cultures of Bronchial Epithelium and Tumor
}

\author{
Fernando Gamarra ${ }^{a}$ Simon Wagnerb Salah Al-Batran ${ }^{a}$ Irmgard Maiera \\ Mirna Castro ${ }^{b}$ Hubert Hautmann $^{a}$ Albrecht Bergnera \\ Reinhold Baumgartnerb Rudolf Maria Hubera \\ aDepartment of Pneumology, Medizinische Klinik Innenstadt, and ' ${ }^{\mathrm{L}}$ Laserforschungslabor an der Urologie, \\ Ludwig Maximilians University of Munich, Germany
}

\section{Key Words}

5-Aminolevulinic acid · Lung

\begin{abstract}
Background: 5-Aminolevulinic acid (5-ALA)-induced protoporphyrin IX (PPIX) fluorescence improves the differentiation of tumor and normal tissue in the bladder, skin and brain. Objective: The kinetics of 5-ALA-induced protoporphyrin IX (PPIX) fluorescence in organ cultures of normal human bronchial epithelium and cocultures of bronchial epithelium and tumor have been studied. Methods: Cultured biopsies of bronchial epithelium were exposed for 5 or $15 \mathrm{~min}$, or continuously to 5-ALA. PPIX fluorescence was quantified for up to $300 \mathrm{~min}$ by spectroscopy. Cocultures of normal bronchial epithelium and a non-small-cell lung cancer cell line (EPLC-32M1) were incubated with 5-ALA. Space-resolved fluorescence microscopy was used to quantify PPIX fluorescence kinetics in the tumor and normal epithelium. Results: In cultures of normal epithelium, PPIX fluorescence kinetics were shown to depend on the duration of exposure to 5-ALA. There was a trend to higher fluorescence intensities with longer exposure times. In cocultures of bronchial epithelium and tumor, increases of
\end{abstract}

fluorescence intensity were significantly greater in the tumor. Best tumor/normal tissue fluorescence ratios were found between 110 and 160 min after exposure to 5-ALA. Conclusion: Data obtained in this coculture system of bronchial epithelium and tumor is valuable to optimize modalities of fluorescence bronchoscopy for the diagnosis of early bronchial carcinoma.

Copyright $\odot 2002$ S. Karger AG, Basel

\section{Introduction}

Life expectancy in patients with lung cancer mainly depends on the tumor stage at the first diagnosis. Only small tumors have a real chance of curative treatment. Moreover, early detection of relapses improves the success of therapy. To improve sensitivity of bronchoscopy for early detection of lung cancer, techniques applying tissue autofluorescence or fluorescent dyes are under development [1]. The application of 5-aminolevulinic acid (5ALA), which leads to intracellular production of the fluorescent protoporphyrin IX (PPIX), shows promising results in early diagnosis and therapy of bladder and skin malignancies [2-5] and the demarcation of brain tumors [6]. The feasibility of inhalative or systemic application of

\begin{tabular}{ll}
\hline KARGER & ( ) 2002 S. Karger AG, Basel \\
Fax +4161306 1234 & $0025-7931 / 02 / 0695-0445 \$ 18.50 / 0$ \\
$\begin{array}{l}\text { E-Mail karger@karger.ch } \\
\text { www.karger.com }\end{array}$ & $\begin{array}{l}\text { Accessible online at: } \\
\text { www.karger.com/journals/res }\end{array}$
\end{tabular}

\begin{tabular}{l}
\hline Prof. Dr. R.M. Huber \\
Medizinische Klinik Innenstadt, Ludwig Maximilians University \\
Ziemssenstrasse 1, D-80336 Munich (Germany) \\
Tel. +49 895160 2590, Fax +49 8951604905 \\
E-Mail huber@medinn.med.uni-muenchen.de
\end{tabular} 
5-ALA for studies in the bronchi has been tested in selected patients $[7,8]$. More data is needed to optimize these diagnostic techniques. The aim of the present studies was, therefore, to study features of 5-Ala-induced kinetics in an organ culture model of human bronchial epithelium and tumor.

\section{Methods}

Organ Cultures of Bronchial Epithelium and Cocultures of Bronchial Epithelium and Tumor

A three-dimensional organ culture system $[9,10]$ which has been shown to maintain different properties of the human respiratory epithelium in vivo was adapted. Biopsies of the left or right upper lobe carina of patients undergoing bronchoscopy were obtained and further cultured. The ethical committee of the University of Munich had approved the procedure. Particulars on culture conditions have been described elsewhere [11]. After a few days the respiratory epithelium grows around the whole biopsy (fig. 1). Thus the threedimensional organ culture system consists of a nucleus of connective tissue which is completely covered by a multilayered respiratory epithelium. The cultured tissue contains a basal membrane, ciliated cells, secretory cells and basal cells [12] and strongly resembles respiratory epithelium in vivo.

14- to 21-day-old organ cultures of normal bronchial epithelium were halved with a scalpel creating a wound in the epithelial surface at one side of each piece (fig. 1). Cells of the non-small-cell lung cancer line EPLC-32M1 [13] (approximately $10^{6}$ cells suspended in $1 \mu \mathrm{l}$ ) were placed in contact with the wounded surface. After 14 days tumor cells had adhered to the wounded surface and infiltrated the stroma of the organ cultures [11].

\section{Application of 5-ALA}

Cultures were incubated in $300 \mathrm{mg} / \mathrm{l}$ 5-ALA (Medac GmbH, Hamburg, Germany) which was dissolved in serum-free, HEPESbuffered DMEM at $37^{\circ} \mathrm{C}$.

\section{5-ALA-Induced PPIX Fluorescence in Normal Bronchial}

Epithelium Depending on the Time of Incubation with 5-ALA

Cultures of 14-day-old normal bronchial epithelium were randomized and incubated for 5 or $15 \mathrm{~min}$, or continuously with 5-ALA ( $\mathrm{n}=9$ /group). The cultures were placed in a specially constructed chamber with controlled temperature $\left(37^{\circ} \mathrm{C}\right.$; Effenberg, Munich, Germany) containing HEPES-buffered DMEM medium for the first two groups, or the 5-ALA solution for the continuously incubated group. PPIX fluorescence was measured at $\mathrm{t}=30,60,90,120,150$, $180,210,240,270$ and 300 min after the beginning of the incubation with 5-ALA. For each measurement the cultures were epi-illuminated with a filtered $(\lambda=375-420 \mathrm{~nm})$ xenon-short arc lamp (D-light, Storz GmbH, Tuttlingen, Germany). Fluorescence emission was collected from the whole surface of the organ culture by a fused silica fiber attached to an intensified optical multichannel analyzer (OSMA, SI Instruments, Gilching, Germany). Fluorescence intensities between 628 and $644 \mathrm{~nm}$ (corresponding to the maximum peak of PPIX fluorescence at $635 \mathrm{~nm}$ ) were integrated after normalizing the spectra to tissue autofluorescence $(590-600 \mathrm{~nm})$. Finally, the values obtained at $\mathrm{t}=35 \mathrm{~min}$, which were considered as baseline, were subtracted.

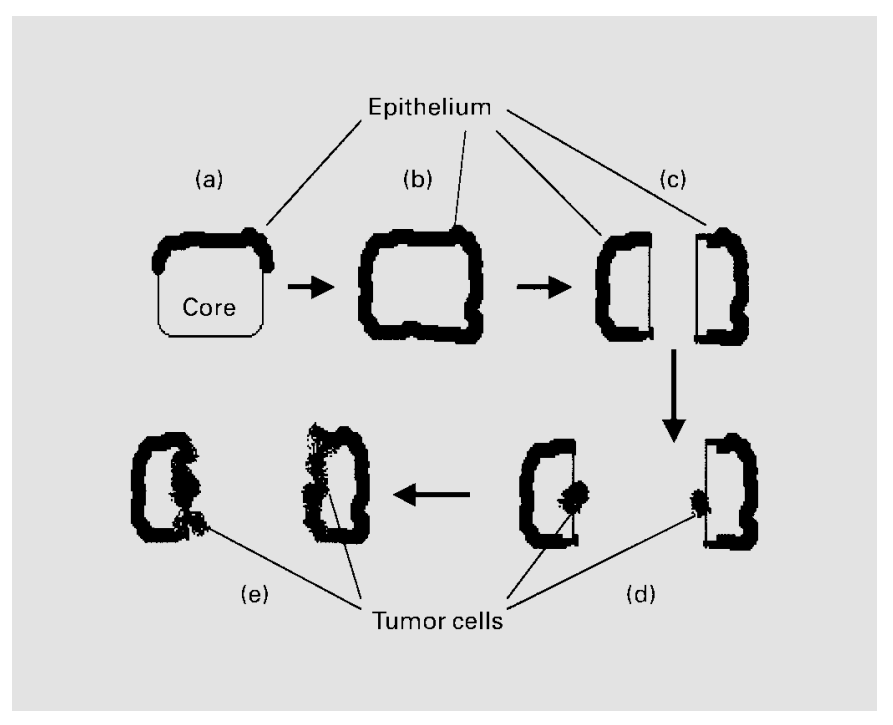

Fig. 1. Schematic drawing illustrating culture techniques. Biopsies of human bronchial epithelium are put in culture (a). Respiratory epithelium grows around the whole biopsy (b). These organ cultures of normal epithelium were used for the first series of experiments. Other cultures were halved after 14 days with a scalpel (c). Tumor cells were implanted on the wounded surface (d) in order to produce cocultures of normal epithelium and tumor (e).

\section{5-ALA-Induced PPIX Fluorescence in Cocultures of Normal Bronchial Epithelium and Tumor}

Cocultures of normal bronchial epithelium and tumor $(n=7)$ were incubated for $15 \mathrm{~min}$ with 5-ALA and then placed under a Zeiss fluorescence microscope. PPIX fluorescence was visualized by epiillumination with a filtered $\mathrm{Hg}$ lamp $(\lambda=405 \mathrm{~nm})$. Fluorescence images $(\lambda>590 \mathrm{~nm})$ were acquired with a CCD camera (Tricam sl 3 -chip-CCD, Storz) at $\mathrm{t}=35,50,65,80,95,110,125,140,160,190$, 220,250 and $280 \mathrm{~min}$ after the beginning of the incubation with 5ALA. Space-resolved quantification of fluorescence intensities was performed off-line (OPTIMAS image analyzing system, Media Cybernetics, Silver Spring, Md., USA) in interactively defined region of interest areas (ROIs): 4 ROIs at the borders of the normal bronchial epithelium, 3 at the borders of the tumor, 1 ROI at the center of normal tissue and 1 at a corresponding central area of the tumor (fig. 2). Care was taken to use similarly thick areas at the tumor and normal epithelium of the borders. Fluorescence values obtained at $\mathrm{t}=35$ min were considered as baseline and subtracted.

Differentiation between tumor-infiltrated and normal bronchial epithelium was accomplished by analyzing an image of the coculture acquired under conventional light microscopy. The following criteria were applied: (1) the normal borders were smooth in contrast to the tumor borders, and (2) the tumor cells had been implanted on the cut edge of the original organ culture of bronchial epithelium. This straight edge could be identified in most of the cocultures. A second observer controlled the position of the tumor with respect to the normal portions. 


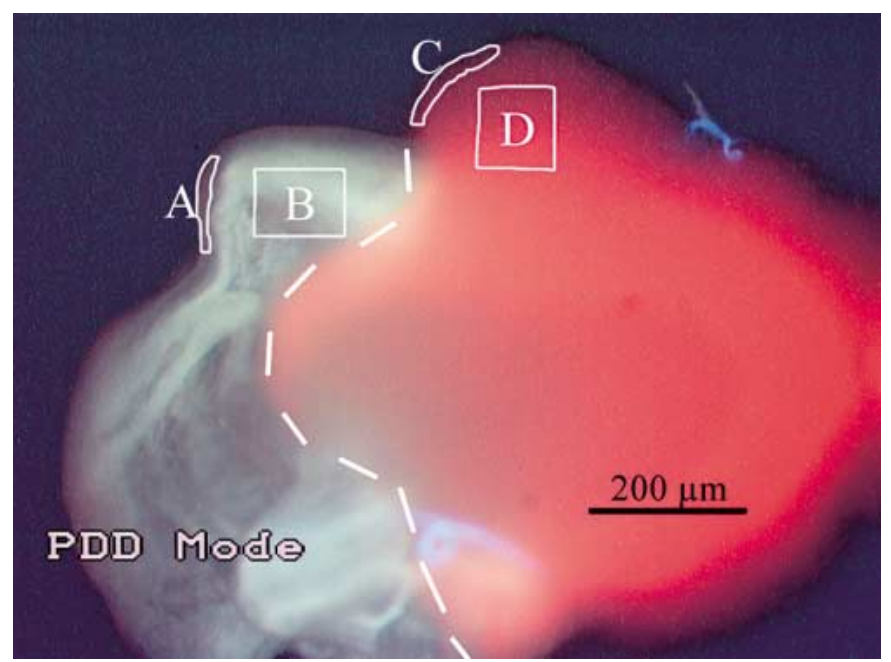

Fig. 2. Digitized fluorescence image of a coculture of bronchial epithelium and tumor $120 \mathrm{~min}$ after the beginning of incubation with 5-ALA (30 mg/l, $15 \mathrm{~min}$ incubation time) showing the defined ROI areas on normal bronchial epithelium at the borders (A), normal epithelium at the center (B), tumor at the borders (C), and tumor at the center of the culture (D) which were used for quantification of fluorescence. The white scattered line indicates the border between tumor (on the right) and normal tissue. The increased red fluorescence intensity (corresponding to PPIX) at the tumor site as compared to normal epithelium can be appreciated.

\section{Statistics}

The Kruskal-Wallis test was used for nonparametric one-way analysis of variance and multiple comparisons of ranks of several independent samples. Single unpaired samples were compared by the Friedman test and paired samples by the Wilcoxon signed rank test. PPIX fluorescence kinetics of tumor and normal epithelium in cocultures were compared using the two-way repeated measures analysis of variance on one-factor balanced design. The SigmaStat statistical software package was used (Jandel Scientific, USA). Data are given as mean \pm standard error of the mean (SE).

\section{Results}

In the first series of experiments, PPIX fluorescence intensities were measured in organ cultures of normal bronchial epithelium by spectroscopy. Analysis of the acquired spectra confirmed that increases of fluorescence after incubation with 5-ALA were due to PPIX. PPIX fluorescence kinetics in the organ cultures of normal bronchial epithelium is depicted in figure 3. Cultures incubated for 5 or 15 min with 5-ALA showed an almost linear increase of fluorescence intensity during the first 80 or

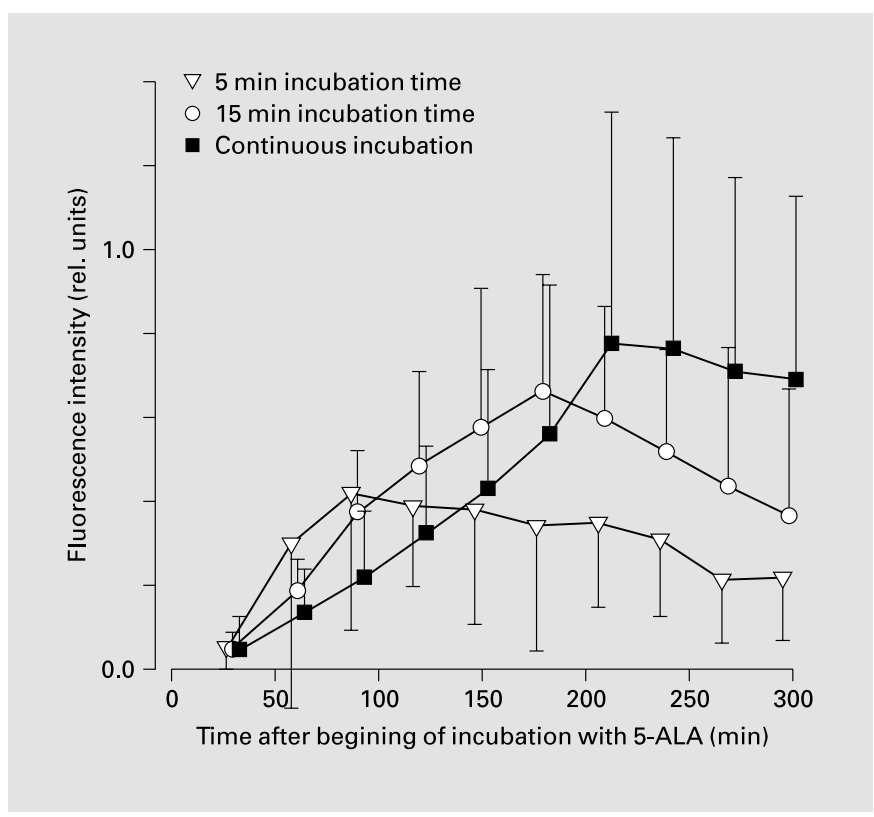

Fig. 3. Diagram shows PPIX fluorescence intensities (arbitrary units; mean $\pm \mathrm{SE} ; \mathrm{n}=9$ /group) measured by spectroscopy in cultures of normal human bronchial epithelium incubated for 5 or $15 \mathrm{~min}$, or continuously with 5-ALA. There is a trend to increased fluorescence intensities with longer incubation times (nonsignificant; two-way repeated measures analysis of variance on one-factor balanced design for comparing kinetics, Kruskal-Wallis test for comparing maximal fluorescence intensities).

$180 \mathrm{~min}$, respectively, and a decrease later. In cultures continuously incubated with 5-ALA, a plateau of PPIX fluorescence began at $220 \mathrm{~min}$ and persisted for the rest of the experiment. Maximal PPIX fluorescence intensities seemed to increase depending on the time of incubation with 5-ALA. The differences between the groups were not statistically significant, however. The time to reach maximal fluorescence intensities was $126.7 \pm 36.8,193.3 \pm$ $37.7 *$ and $236.7 \pm 51.9 *$ min (mean $\pm \mathrm{SE}$ ) in the cultures incubated for 5 or $15 \mathrm{~min}$, or continuously with 5-ALA, respectively $(* \mathrm{p}<0.05$ vs. the other groups; Kruskal-Wallis test). These significant differences support the dependency of PPIX fluorescence kinetics from the time of incubation with 5-ALA.

Cocultures of tumor and normal bronchial epithelium had been exposed for $15 \mathrm{~min}$ to 5-ALA. The first measurement of fluorescence intensity at $t=35 \mathrm{~min}$ did not show significant differences between normal epithelium and tumor in the ROIs at the borders of the cultures. In the ROIs positioned at the central portions, significantly higher fluorescence intensities were measured in the nor- 


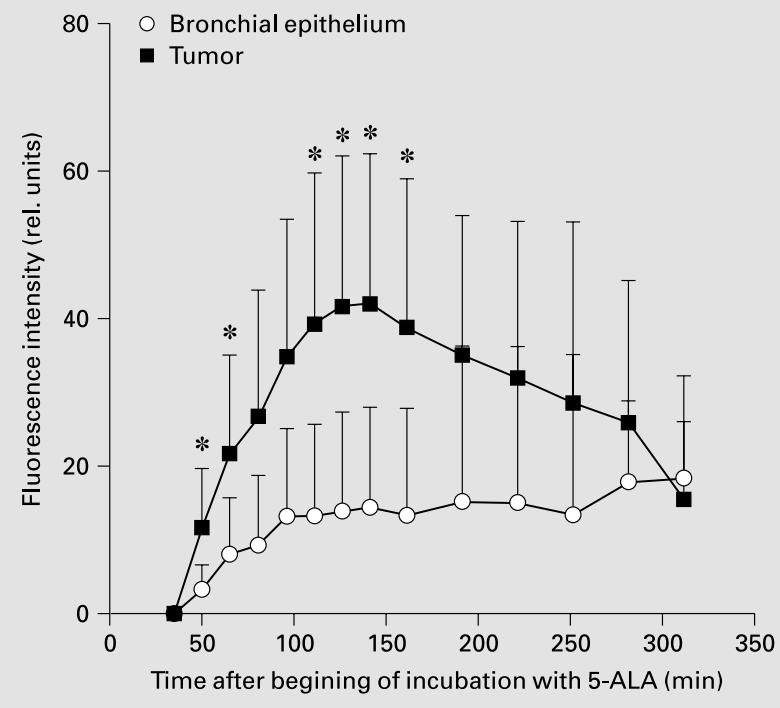

Fig. 4. Diagram shows kinetics of PPIX fluorescence intensities in normal human bronchial epithelium and tumor after incubation with 5-ALA for $15 \min (n=7$; values are mean \pm SE) as measured in ROIs at the borders of cocultures. Kinetics differed significantly if the values were corrected to $t=35 \mathrm{~min}$ as baseline $(\mathrm{p}<0.05$, two-way repeated measures analysis of variance on one-factor balanced design). $* \mathrm{p}<0.05$ for fluorescence intensity in the tumor versus corresponding values in normal bronchial epithelium (Wilcoxon signed rank test for paired samples).

mal tissue as compared to the tumor at the first measurement (table 1). Later on there was a more pronounced increase of PPIX fluorescence intensities in the tumor as compared to normal tissue either at the borders or at the center of the culture (fig. 4). If the fluorescence values were corrected to the values at $\mathrm{t}=35 \mathrm{~min}$ as baseline, statistical significant differences of PPIX fluorescence kinetics between tumor and normal epithelium were demonstrated. There were no significant differences of kinetics between normal epithelium at the center versus normal epithelium at the borders, or between tumor tissues at the center versus tumor at the borders (two-way repeated measures analysis of variance on one factor design; data not shown in detail). At the borders, uncorrected tumor fluorescence intensities were significantly higher than in the corresponding normal epithelium at 50 and $65 \mathrm{~min}$, and between 110 and $160 \mathrm{~min}$ (fig. 4). The time that was needed to reach maximal PPIX fluorescence intensities in the ROIs at the borders was $167.86 \pm 117.2$ or $225.0 \pm$
Table 1. Fluorescence intensities in ROI

\begin{tabular}{ll}
\hline ROI & Fluorescence intensity \\
\hline Normal epithelium, border & $37.24 \pm 19.13$ \\
Normal epithelium, center & $74.27 \pm 49.83^{*}$ \\
Tumor border & $41.04 \pm 17.24$ \\
Tumor center & $46.78 \pm 21.22$ \\
\hline
\end{tabular}

Baseline fluorescence intensities $(t=35$ min after the beginning of incubation with 5-ALA) in cocultures of bronchial epithelium and tumor (mean $\pm \mathrm{SE} ; \mathrm{n}=7$ ).

$* \mathrm{p}<0.05$ vs. the other groups (Kruskal-Wallis test).

83.54 for tumor or normal epithelium, respectively (mean $\pm \mathrm{SE} ; \mathrm{n}=7$ ). Thus, the borders of the tumor tended to reach maximal fluorescence earlier than those of normal epithelium. The differences were, however, not statistically significant (Kruskal-Wallis test).

\section{Discussion}

Our aim was to study kinetics of 5-ALA-induced PPIX fluorescence in an in vitro model of human bronchial epithelium and infiltrating tumor. An exogenous supply of 5-ALA in excess bypasses the biological regulatory mechanisms leading to the intracellular accumulation of PPIX: the immediate precursor of heme, which can be detected by fluorescence techniques [4]. Topical and systemic application of 5-ALA has been demonstrated to induce greater PPIX fluorescence intensities in tumors as compared to normal tissue in different organs $[2,4,14,15]$.

Patterns of PPIX fluorescence in bronchial epithelium are expected to depend on the distribution of the dye in different cell types, the arrangement of the cells and threedimensional fluorescence scattering. These characteristics are better recreated in organ cultures of bronchial epithelium than monolayer culture systems. Moreover, the possibility of using cocultures of normal epithelium and tumor permits to compared fluorescence intensities and kinetics of both tissue types directly.

One of the main disadvantages of organ cultures is, however, that cell composition and arrangement, thickness of the cultured bronchial epithelium and size of the biopsies are not standardized. Variability of size and quality (i.e. cell composition and arrangement) of single organ cultures may have influenced the fluorescence intensity 
values in the first series of experiments using fluorescence spectroscopy. This technique assessed the sum of fluorescence in the whole culture. Culture size was probably not that relevant in the second series using space-resolved fluorescence microscopy, especially if the measurements were confined to the borders of the organ cultures. The variability of fluorescence in normal bronchial epithelium detected here probably reflects morphological differences of the epithelium. As shown for the cell composition of primary cultures of respiratory epithelium [16], the occurrence of bronchitis or dysplasia in the bronchial epithelium of the donor might affect the quality of the cultured tissue. To reduce variability, we used solely biopsies of the upper lobe carina, selected cultures of a similar size and cultures presenting ciliary beating in at least $50 \%$ of the borders of normal epithelium, as assessed by inverse microscopy. Despite this, standard deviations of fluorescence measurements were relatively high, which is one of the limitations of this study.

It has been shown that rising concentrations of 5-ALA induce increasing PPIX fluorescence intensities and longer times to reach maximal fluorescence in different tissues [17]. We also showed that PPIX fluorescence kinetics depend on the time of exposure to 5-ALA in cultures of normal bronchial epithelium. PPIX fluorescence kinetics might also depend on the application modalities of 5-ALA [18]. The results of our experiments may better apply to topical application of 5-ALA (for example inhalation of 5-ALA), since the patterns of 5-ALA transport by blood are not taken into consideration in our system. The trend to increased maximal fluorescence intensities with longer 5-ALA incubation times suggests that a time-dependent 'reservoir' of PPIX precursors is built in the cell during the incubation with the substance. The contact time with 5-ALA might, therefore, be relevant, in addition to 5-ALA concentration, if the substance is topically applied in the bronchi. The patterns of PPIX kinetics in bronchial tissue after application of 5-ALA we found are similar to those in the gastric and colon mucosa [19]. Studies of PPIX fluorescence kinetics in murine lungs (not specifically bronchial tissue) and tumors have found maximal fluorescence intensities at $180 \mathrm{~min}$ in both of them [20].

We have studied the interaction of EPLC-32M1 tumor cells and organ cultures of human bronchial epithelium by histology and electron microscopy in a previous work [11]. EPLC-32M1 cells have been shown to attach to the wounded side of organ cultures and to infiltrate the acellular core. The tumor cells do not spread into the bronchial epithelium. 14-day-old cocultures as used for the fluorescence experiments did not show evidence of tumor necro- sis. The measurements of PPIX fluorescence in cocultures of normal bronchial epithelium and tumor were performed in ROI areas at the center or at the borders of the culture with the microscope focused at the borders. We considered the nearly spherical form of the organ cultures and assumed that fluorescence values measured at the borders most likely correspond to a defined volume of vital cells and, therefore, are better suited for our studies. The values at the center corresponded to a less welldefined volume, which might include fluorescence of vital cells, of the acellular core of the organ cultures and scattering fluorescence of the surrounding structures. Thus the higher baseline fluorescence intensities measured in the ROIs corresponding to 'normal tissue' at the center (table 1) are probably determined by the high levels of autofluorescence of the acellular nucleus under the epithelium. Tumor cells infiltrating the acellular core of the organ culture probably determine the lower baseline fluorescence intensities in the tumor ROIs at the center. Similar conditions have been proposed as an explanation for the phenomenon of tumor detection by autofluorescence bronchoscopy.

The patterns of PPIX fluorescence kinetics in tumor and normal tissues were similar, with a more pronounced increase of fluorescence in the tumor. Assuming that the volume of tumor and normal tissues at the borders assessed by the measurements should be comparable, the higher PPIX fluorescence intensities in the tumor probably means increased synthesis of PPIX in tumor cells compared to normal bronchial epithelium, as it has been shown for other tissues $[2,4,14,15]$.

These results demonstrate the feasibility of using tumor-infiltrated organ cultures of bronchial epithelium to obtain data on fluorescence of photodynamic dyes in the bronchial system and bronchial carcinoma. The first series showed the importance of the contact time with 5ALA for PPIX fluorescence kinetics. Although normal bronchial epithelial cells also synthesized PPIX, increased fluorescence intensities in the EPLC tumor cells indicate that PPIX might be an indicator of malignancy in the bronchial system. Studies on tumor-infiltrated organ cultures may help to optimize the procedures for fluorescence diagnosis in patients. According to the present results, highest tumor/normal tissue ratios should be expected at about 120-150 min after application of 5-ALA. Further experiments will show if this timing also applies to other tumor types. Bronchoscopic measurements in patients are necessary to confirm this data. In theory, uptake and metabolism of 5-ALA to PPIX could be influenced pharmacologically. The coculture system 
could be used to test whether any treatment improves fluorescence ratios between tumor and normal tissues and consequently the diagnostic value of 5-ALA in the bronchial system.

\section{Acknowledgments}

This study was supported by grants of the Wilhelm Sander Foundation and the Bundesministerium für Bildung und Forschung (BMBF).

\section{References}

1 Lam S, Becker HD: Future diagnostic procedures. Chest Surg Clin N Am 1996;6:363-380.

2 Kriegmair M, Baumgartner R, Knuechel R, Steinbach P, Ehsan A, Lumper W, Hofstadter F, Hofstetter A: Fluorescence photodetection of neoplastic urothelial lesions following intravesical instillation of 5-aminolevulinic acid. Urology 1994;44:836-841.

3 Kriegmair M, Baumgartner R, Lumper W, Waidelich R, Hofstetter A: Early clinical experience with 5-aminolevulinic acid for the photodynamic therapy of superficial bladder cancer. Br J Urol 1996;77:667-671.

4 Kennedy JC, Pottier RH, Pross DC: Photodynamic therapy with endogenous protoporphyrin IX: Basic principles and present clinical experience. J Photochem Photobiol B 1990;6/ 1-2:143-148.

5 Fritsch C, Goerz G, Ruzicka T: Photodynamic therapy in dermatology. Arch Dermatol 1998; 134/2:207-214.

6 Stummer W, Stocker S, Wagner S, Stepp H, Fritsch C, Goetz C, Goetz AE, Kiefmann R, Reulen $\mathrm{HJ}$ : Intraoperative detection of malignant gliomas by 5-aminolevulinic acid-induced porphyrin fluorescence. Neurosurgery 1998; 42:518-525.

7 Huber RM, Gamarra F, Hautmann H, Haussinger K, Wagner S, Castro M, Baumgartner R: 5-Aminolaevulinic acid (ALA) for the fluorescence detection of bronchial tumors. Diagn Ther Endosc 1999;5:113-118.

8 Baumgartner R, Huber RM, Schulz H, Stepp H, Rick K, Gamarra F, Leberig A, Roth C: Inhalation of 5-aminolevulinic acid: A new technique for fluorescence detection of early stage lung cancer. J Photochem Photobiol B 1996;36/2:169-174
9 Steinsvag SK, Strand M, Berg O, Miaguchi M, Olofsson $\mathrm{J}$ : Human respiratory mucosa in a nonadhesive stationary organ culture system. Laryngoscope 1991;101:1323-1331.

10 Fjellbirkeland L, Bjerkvig R, Steinsvag SK, Laerum OD: Nonadhesive stationary organ culture of human bronchial mucosa. Am J Respir Cell Mol Biol 1996;15/2:197-206.

11 Al-Batran S, Astner ST, Supthut M, Gamarra F, Brueckner K, Welsch U, Knuechel R, Huber RM: Three-dimensional in vitro cocultivation of lung carcinoma cells with human bronchial organ culture as a model for bronchial carcinoma. Am J Respir Cell Mol Biol 1999;21/2:200208.

12 Bals R, Gamarra F, Kaps A, Grundler S, Huber RM, Welsch U: Secretory cell types and cell proliferation of human bronchial epithelial cells in an organ-culture system. Cell Tissue Res 1998;293:573-577.

13 Bepler G, Koehler A, Kiefer P, Havemann K, Beisenherz K, Jaques G, Gropp C, Haeder M: Characterization of the state of differentiation of six newly established human non-small-cell lung cancer cell lines. Differentiation 1988;37/ 2:158-171.

14 Fritsch C, Batz J, Bolsen K, Schulte KW, Zumdick M. Ruzicka T, Goerz G: Ex vivo application of delta-aminolevulinic acid induces high and specific porphyrin levels in human skin tumors: Possible basis for selective photodynamic therapy. Photochem Photobiol 1997;66/ $1: 114-118$
15 Regula J, MacRobert AJ, Gorchein A, Buonaccorsi GA, Thorpe SM, Spencer GM, Hatfield AR, Bown SG: Photosensitisation and photodynamic therapy of oesophageal, duodenal, and colorectal tumours using 5 aminolaevulinic acid induced protophorphyrin IX: A pilot study. Gut 1995;36/1:67-75.

16 Franklin WA, Folkvord JM, Varella Garcia M, Kennedy T, Proudfoot S, Cook R, Dempsey EC, Helm K, Bunn PA, Miller YE: Expansion of bronchial epithelial cell populations by in vitro culture of explants from dysplastic and histologically normal sites. Am J Respir Cell Mol Biol 1996;15/3:297-304.

17 van der Veen N, van Leengoed HL, Star WM: In vivo fluorescence kinetics and photodynamic therapy using 5-aminolaevulinic acidinduced porphyrin: Increased damage after multiple irradiations. Br J Cancer 1994;70: 867-872.

18 van der Veen N, Bruijn HS, Berg RJ, Star WM: Kinetics and localisation of PPIX fluorescence after topical and systemic ALA application, observed in skin and skin tumours of UVBtreated mice. Br J Cancer 1996;73:925-930.

19 Loh CS, Vernon D, MacRobert AJ, Bedwell J, Bown SG, Brown SB: Endogenous porphyrin distribution induced by 5 -aminolaevulinic acid in the tissue layers of the gastrointestinal tract. J Photochem Photobiol B 1993;20/1:47-54.

20 Campbell DL, Gudgin Dickson EF, Forkert PG, Pottier RH, Kennedy JC: Detection of early stages of carcinogenesis in adenomas of murine lung by 5-aminolevulinic acid-induced protoporphyrin IX fluorescence. Photochem Photobiol 1996;64:676-682. 